Image

\title{
Une hypergammaglobulinémie considérable
}

\section{A huge hypergammaglobulinemia}

\author{
F. Herrera, S. Duband, M. Koenig, P. Cathébras* \\ Service de médecine interne, hôpital Nord, 42055 Saint-Étienne cedex 02, France \\ Disponible sur Internet le 26 juin 2007
}

Mots clés : Hypergammaglobulinémie ; Leishmaniose viscérale

Keywords: Hypergammaglobulinemia; Visceral leishmaniasis

\section{Histoire}

Un homme de 22 ans était adressé en médecine interne pour le bilan d'une fièvre prolongée avec frissons, altération de l'état général et pesanteur de l'hypochondre droit, évoluant depuis six mois environ. Son seul antécédent remarquable était la notion d'une splénectomie à l'âge de sept ans pour un abcès splénique à Salmonella sp. Les examens faits en ville avaient révélé une cytolyse modérée (transaminases à $2 \mathrm{~N}$ ) et une lymphocytose (5 à $6 \mathrm{G} / \mathrm{l})$. L'examen révélait une micropolyadénopathie généralisée et une hépatomégalie sensible. Les examens biologiques initiaux montraient un taux d'hémoglobine à $12 \mathrm{~g} / \mathrm{dl}$, des lymphocytes à $4 \mathrm{G} / 1$ et des monocytes à $2 \mathrm{G} / \mathrm{l}$. Les plaquettes et l'hémostase étaient normales, la créatininémie à $69 \mu \mathrm{mol} / 1$, la protéine $\mathrm{C}$ réactive à $20 \mathrm{mg} / \mathrm{l}$, les ALAT/ALAT à $8 \mathrm{~N}$, les phosphatases alcalines à $4 \mathrm{~N}$, les $\mathrm{LDH}$ à $3 \mathrm{~N}$, les triglycérides à $5 \mathrm{~g} / \mathrm{l}$, les gammaglobulines à $60 \mathrm{~g} / \mathrm{l}$ sur l'électrophorèse des protéines (Fig. 1). De nombreuses perturbations immunologiques étaient détectées avec un test de Coombs positif de type $\operatorname{IgG}$, une présence d'anticorps antinucléaires (1/80 mouchetés) et d'anticorps antimuscles lisses de type antiactine (1/160), un facteur rhumatoïde $\operatorname{IgG}$, et une présence de cryoglobuline non typée. L'imagerie ne révélait qu'une hépatomégalie homogène. La sérologie du VIH était négative et aucun facteur d'immunodépression n'était retrouvé en dehors de la splénectomie.

\footnotetext{
* Auteur correspondant.

Adresse e-mail : pascal.cathebras@chu-st-etienne.fr (P. Cathébras).
}

\section{Diagnostic}

Une leishmaniose viscérale.

\section{Commentaires}

Le myélogramme du patient était riche, granuleux neutrophile, avec présence de macrophages contenant de nombreuses leishmanies et d'un syndrome d'activation macrophagique (Fig. 2). La leishmanie était identifiée comme Leishmania infantum zymodème NOM-1. L'évolution clinique et biologique était rapidement favorable sous amphotéricine B liposomale.

Une hypergammaglobulinémie polyclonale évoque avant tout pour l'interniste une connectivite (syndrome de GougerotSjögren en particulier), une sarcoïdose, une hépatopathie chronique, mais certaines causes infectieuses doivent être envisagées : bactériennes (par exemple tuberculose, bartonnellose), virales (en particulier VIH et CMV), et parasitaires (mycoses profondes, paludisme, toxoplasmose, leishmaniose viscérale). Ce dernier diagnostic est en général évoqué devant une fièvre hectique associée à une splénomégalie et à une pancytopénie, deux symptômes qui manquaient dans notre observation en raison de la splénectomie.

L'asplénie ne semble pas représenter un facteur de risque significatif pour la leishmaniose, au contraire de l'infection à VIH [1] ou d'autres causes d'immunodépression comme la corticothérapie au long cours [2]. Nous n'avons retrouvé que d'exceptionnelles observations de leishmaniose viscérale survenant chez des sujets splénectomisés [3,4]. En revanche, on peut rappeler qu'une autre protozoose, la babésiose, dans sa forme européenne (Babesia divergens), est observée chez l'homme quasi électivement chez des sujets splénectomisés[5]. 


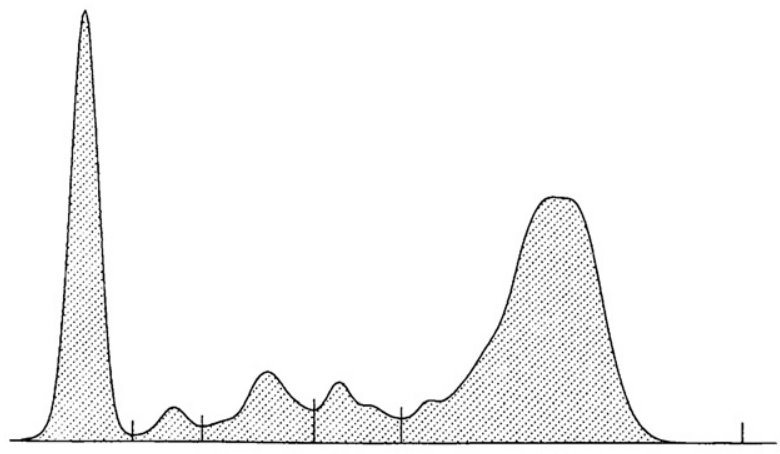

Fig. 1. Électrophorèse des protides : hypergammaglobulinémie majeure.

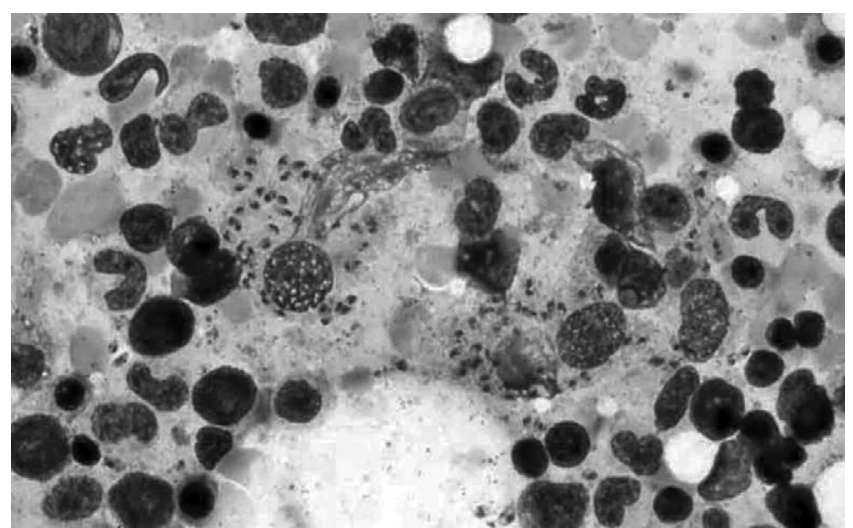

Fig. 2. Myélogramme: macrophages contenant de nombreuses leishmanies, syndrome d'activation macrophagique.

\section{Références}

[1] Paredes R, Munoz J, Diaz I, Domingo P, Gurgui M, Clotet B. Leishmaniasis in HIV infection. J Postgrad Med 2003;49:39-49.

[2] Pittalis S, Nicastri E, Spinazzola F, Ghirga P, De Marco M, Paglia MG, et al. Leishmania infantum leishmaniasis in corticosteroid-treated patients. BMC Infect Dis 2006;6:177.

[3] Bada JL, Arderiu A, Gimenez J, Gumez-Acha JA. Kala-azar of long-standing evolution in an asplenic patient. Trans R Soc Trop Med Hyg 1979;73:347-8.

[4] Pavone P, Giugno GR, Luca G, Samperi P, Consalvo C, Lucenti C. Leishmaniasis in a splenectomized thalassodrepanocytic young adult. Minerva Pediatr 2005;57:325-8.

[5] Meliani P, Khatibi S, Randazzo S, Gorenflot A, Marchou B. Babésioses humaines. Med Mal Infect 2006;36:499-504. 